\title{
Topographic changes in cerebral blood flow and reduced white matter integrity in the first 2 weeks following revascularization surgery in adult moyamoya disease
}

\author{
Ken Kazumata, MD, PhD, ${ }^{1}$ Khin Khin Tha, MD, PhD, ${ }^{2}$ Haruto Uchino, MD, ${ }^{1}$ Tohru Shiga, MD, PhD, ${ }^{3}$ \\ Hideo Shichinohe, MD, PhD, ${ }^{1}$ Masaki Ito, MD, PhD, ${ }^{1}$ Naoki Nakayama, MD, PhD, ${ }^{1}$ and \\ Takeo Abumiya, MD, PhD'
}

'Departments of Neurosurgery, ${ }^{2}$ Radiobiology and Medical Engineering, and ${ }^{3}$ Nuclear Medicine, Hokkaido University Graduate School of Medicine, Sapporo, Japan

\begin{abstract}
OBJECTIVE After revascularization surgery, hyperperfusion and ischemia are associated with morbidity and mortality in adult moyamoya disease (MMD). However, structural changes within the brain following revascularization surgery, especially in the early postsurgical period, have not been thoroughly studied. Such knowledge may enable improved monitoring and clinical management of hyperperfusion and ischemia in MMD. Thus, the objective of this study was to investigate the topographic and temporal profiles of cerebral perfusion and related white matter microstructural changes following revascularization surgery in adult MMD.
\end{abstract}

METHODS The authors analyzed 20 consecutive surgeries performed in 17 adults. Diffusion imaging in parallel with serial measurements of regional cerebral blood flow (rCBF) using SPECT was performed. Both voxel-based and regionof-interest analyses were performed, comparing neuroimaging parameters of postoperative hemispheres with those of preoperative hemispheres at 4 different time points within 2 weeks after surgery.

RESULTS Voxel-based analysis showed a distinct topographic pattern of cerebral perfusion, characterized by increased $\mathrm{rCBF}$ in the basal ganglia for the first several days and gradually increased $\mathrm{rCBF}$ in the lateral prefrontal cortex over 1 week ( $p<0.001)$. Decreased rCBF was also observed in the lateral prefrontal cortex, occipital lobe, and cerebellum contralateral to the surgical hemisphere $(p<0.001)$. Reduced fractional anisotropy $(F A)$ and axial diffusivity $(A D)$, as well as increased radial diffusivity (RD), were demonstrated in both the anterior and posterior limbs of the internal capsule ( $p$ $<0.001$ ). Diffusion parameters demonstrated the greatest changes in both FA and RD on Days 1-2 and in AD on Days 3-6; FA, RD, and AD recovered to preoperative levels on Day 14. Patients with transient neurological deteriorations (TNDs), as compared with those without, demonstrated greater increases in $\mathrm{rCBF}$ in both the lateral prefrontal cortex and striatum as well as smaller FAs in the posterior limb of the internal capsule $(p<0.05)$.

CONCLUSIONS The excessively increased $\mathrm{rCBF}$ and the recovery process were heterogeneous across brain regions, demonstrating a distinct topographic pattern during the initial 2 weeks following revascularization surgery in MMD. Temporary impairments in the deep white matter tract and immediate postoperative ischemia were also identified. The study results characterized postoperative brain perfusion as well as the impact of revascularization surgery on the brain microstructure. Notably, rCBF and white matter changes correlated to TNDs, suggesting that these changes represent potential neuroimaging markers for tracking tissue structural changes associated with hyperperfusion during the acute postoperative period following revascularization surgery for MMD.

https://thejns.org/doi/abs/10.3171/2016.6.JNS16653

KEY WORDS moyamoya disease; hyperperfusion; regional cerebral blood flow; diffusion tensor imaging; white matter; statistical parametric mapping; vascular disorders

\footnotetext{
ABBREVIATIONS $A D=$ axial diffusivity; DKI = diffusion kurtosis imaging; $D T I=$ diffusion tensor imaging; $F A$ = fractional anisotropy; MAP = mean arterial pressure; $M C A$ = middle cerebral artery; $\mathrm{MK}=$ mean kurtosis; $\mathrm{MMD}=$ moyamoya disease; $\mathrm{MNI}=$ Montreal Neurological Institute; MPRAGE = magnetization-prepared rapid gradient-echo; rCBF = regional cerebral blood flow; RD = radial diffusivity; RK = radial kurtosis; ROI = region of interest; SPM8 = Statistical Parametric Mapping version 8; STA = superficial temporal artery; TND $=$ transient neurological deterioration.
}

SUBMITTED March 15, 2016. ACCEPTED June 8, 2016.

INCLUDE WHEN CITING Published online September 2, 2016; DOI: 10.3171/2016.6.JNS16653. 
$\mathrm{M}$ OYAMOYA disease (MMD) is characterized by the compensatory development of enlarged and weak basal perforating arteries (moyamoya vessels) in response to bilateral occlusive changes in the internal carotid system. ${ }^{23}$ Although revascularization surgery improves chronic ischemia in MMD several months after the surgery,${ }^{14}$ cerebral hemodynamics may be unstable for the first several weeks. ${ }^{26}$ Hyperperfusion and ischemia are closely associated with poor prognoses after revascularization surgery. ${ }^{8,919}$ However, the temporal profile of regional cerebral blood flow (rCBF) alterations has not been sufficiently investigated in detail during the initial 2 weeks after surgery. $2,8,12,35$ Identifying characteristics of the processes and effects of hyperperfusion and ischemia after surgery, especially in the early postsurgical period, will allow for improved monitoring and treatment of these conditions.

While revascularization surgery in MMD potentially improves cognitive performance, brain damage due to postoperative hyperperfusion can impair cognitive function or restrict improvement. ${ }^{32}$ Previous investigation revealed impaired white matter integrity in the hemisphere exposed to hyperperfusion after carotid endarterectomy. ${ }^{28}$ In revascularization surgery for MMD, gray matter lesions are transiently found on conventional MRI. ${ }^{13,16}$ Subcortical edema has also been reported, although it is not frequently found in the majority of adult patients. ${ }^{33}$ Whether the revascularization procedure in MMD induces covert structural changes remains unclear. In MMD, both diffusion tensor imaging (DTI) and diffusion kurtosis imaging (DKI) have provided in vivo measurements of microstructural changes in white matter that appeared normal on conventional MRI. ${ }^{20,21}$ Thus, it is speculated that diffusion MRI may be sensitive to subtle microstructural changes following revascularization surgery in MMD.

Voxel-based statistical analysis allows comparisons of neuroimaging parameters across different subjects as well as different conditions. ${ }^{25}$ The main objectives of the present study were to investigate the changes in $\mathrm{rCBF}$ in parallel with white matter integrity through voxel-based analysis. We also investigated whether $\mathrm{rCBF}$ in the early phase was associated with hyperperfusion and postoperative neurological symptoms. The management of systemic blood pressure is controversial because of coexisting chronic ischemia. ${ }^{9,26}$ Accordingly, the association between systemic blood pressure and postoperative rCBF alterations was also explored.

\section{Methods \\ Participants}

This prospective study was approved by the Research Ethics Committee of Hokkaido University Hospital. Written informed consent was obtained from all participants. Twenty consecutive revascularization surgeries were performed in 17 patients, 5 men and 12 women, with a mean age of $37.1 \pm 10.6$ years (range 19-50 years). The study period was January 2013 through December 2015. Study inclusion criteria were a clinical diagnosis of idiopathic MMD (according to the consensus criteria and guidelines for MMD proposed by the Research Committee on Spontaneous Occlusion of the Circle of Willis) and an age $>18$ years. ${ }^{23}$

\section{Surgical Procedure and Perioperative Management}

The surgical procedure consisted of double superficial temporal artery-middle cerebral artery (STA-MCA) anastomosis. In this procedure, after a large frontotemporal craniotomy, the dura mater is opened while preserving the middle meningeal artery. Both the parietal and frontal branches of the STA are anastomosed to the cortical branch of the MCA. Direct anastomosis is generally performed in the prefrontal region as well as on the superior temporal gyrus. The dural flap is inverted and placed on the surface of the brain to increase collateral formation. The temporal muscle is then placed on the surface of the brain, completing encephaloduroarteriosynangiosis. ${ }^{17,22}$

Antiplatelet administration in patients with ischemic onset (transient ischemic attack or cerebral infarction) was discontinued at least 1 week prior to the surgery. Every patient received $60 \mathrm{mg}$ of edaravone, a free oxygen radical scavenger, with $100 \mathrm{ml}$ of normal saline (Tanabe Mitsubishi Pharma Co.) at the beginning of the surgery. The edaravone $(30 \mathrm{mg}$ ) was administered twice daily for 7 postsurgical days. Continuous elevated systolic blood pressure above $140 \mathrm{~mm} \mathrm{Hg}$ was treated with diltiazem hydrochloride administered intravenously by continuous infusion.

\section{Study Protocol and Image Acquisition}

The patients serially underwent both SPECT and MRI studies. Regional CBF was measured using ${ }^{123} \mathrm{I}-\mathrm{N}$-isopropyl$p$-iodoamphetamine (IMP) SPECT with a SPECT scanner (GCA-9300R, Toshiba Medical Systems Corp. Ltd.). Magnetic resonance imaging studies included structural MRI (magnetization-prepared rapid gradient-echo [MPRAGE]) and DTI/DKI, performed with a 3.0-T imager (Achieva TX, Philips Medical Solutions). The first scans were obtained before the revascularization surgery. Postoperative followup with both SPECT and MRI was scheduled for 4 times: Days 1-2, Days 3-6, Days 7-8, and Days 11-14. Postoperative SPECT studies were scheduled on Day 0 in some of the patients, who then underwent a second SPECT study on Day 2. In patients with stable postoperative courses, SPECT on postoperative Days 11-14 might be cancelled at the discretion of the physician responsible for the treatment. The SPECT and MRI studies that interfered with postoperative management were canceled and rescheduled. As a result, 62 SPECT measurements were obtained during the postoperative period as follows ( $\mathrm{n}$ represents the number of measurements): Day 0, $\mathrm{n}=9$; Days 1-2, $\mathrm{n}=19$; Days 3-6, $\mathrm{n}=11$; Days 7-8, $\mathrm{n}=17$; and Days $11-14, \mathrm{n}=6$. Seventythree MRI studies were performed as follows (n represents the number of studies): Days $1-2, \mathrm{n}=18$; Days $3-6, \mathrm{n}=17$; Days 7-8, $\mathrm{n}=19$; and Days 11-14, $\mathrm{n}=19$.

The scan parameters for DKI were as follows: TR $5051 \mathrm{msec}$; TE $85 \mathrm{msec}$; flip angle $90^{\circ}$; FOV $224 \times 224$ $\mathrm{mm}^{2}$; matrix size $128 \times 128$; b values 0,1000 , and 2000 $\mathrm{s} / \mathrm{mm}^{2}$; number of diffusion gradient directions 32 ; slice thickness $3 \mathrm{~mm}$; interslice gap $0 \mathrm{~mm}$; number of slices 43; and number of excitations 1 . The 3D MPRAGE was performed with TR $6.8 \mathrm{msec}$, TE $3.1 \mathrm{msec}$, flip angle $8^{\circ}$, and TI $1100 \mathrm{msec}$. The scan parameters for T2-weighted imaging were TR $4137 \mathrm{msec}$, TE $90 \mathrm{msec}$, and effective echo train length 15, and those for FLAIR imaging were TR $10000 \mathrm{msec}$, TR $100 \mathrm{msec}$, and TI $2700 \mathrm{msec}$. Axial 
fast spin echo T2-weighted and FLAIR images were used to diagnose cortical gray matter swelling. ${ }^{13}$

\section{Postprocessing Imaging Data SPECT}

The SPECT images were spatially normalized to the standard SPECT template in Statistical Parametric Mapping version 8 (SPM8; Wellcome Department of Cognitive Neurology, University College London, London, UK; http://www.fil.ion.ucl.ac.uk/spm/) and smoothed with a 14-mm full width at half maximum gaussian kernel.

\section{MRI}

Registration between the echo planar images (EPIs) with no diffusion weighting $\left(b=0 \mathrm{~s} / \mathrm{mm}^{2}\right)$ and the corresponding DTI/DKI data as well as correction for eddy current distortion was performed at the MRI operator console. The diffusion imaging data were processed using MATLAB R2015a (MathWorks) and Diffusional Kurtosis Estimator (http://nitrc.org/projects/dke). ${ }^{34}$ Seven DKI and DTI parameters were extracted from the Diffusion Kurtosis Estimator: mean kurtosis (MK), radial kurtosis (RK), axial kurtosis, fractional anisotropy (FA), mean diffusivity, radial diffusivity (RD), and axial diffusivity (AD).

Both voxel-based and region of interest (ROI)based analyses were performed. First, each subject's 3D MPRAGE images were coregistered to the $b=0$ EPIs using the FLIRT algorithm in FSL (http://fsl.fmrib.ox.ac.uk/ fsl/fslwiki/). The coregistered 3D MPRAGE images were normalized to the ICBM152 template by affine transformation, and the transformation matrices were applied to each diffusion parameter image in SPM8. Spatial smoothing was applied with a full width at half maximum of $6 \mathrm{~mm}$.

\section{Data Analysis}

Both postoperative SPECT and diffusion MR images were divided into 5 categories: postoperative Day 0 (SPECT only), Days 1-2, Days 3-6, Days 7-8, and Days 11-14. Alterations in $\mathrm{rCBF}$ and diffusion parameters in the postoperative periods were compared voxel by voxel with each individual's corresponding preoperative images using the paired t-test model in SPM8. To explore the differences, statistical significance was set at $p<0.001$ without correction for family-wise error. Clusters showing statistical significance and containing more than 50 voxels were considered as areas with significant changes. Images obtained in patients with left revascularization were flipped to the right side, and the hemisphere with revascularization was treated as the right hemisphere. Region of interest-based analysis was also performed to extract the $\mathrm{rCBF}$, DKI, and DTI parameters from the images in the standardized space. After exploratory voxel-by-voxel analysis, ROIs were defined in areas with significant changes. Two ROIs were chosen in the SPECT analysis: one in the striatum and one in the lateral prefrontal cortex. Regions of interest were defined using a template available as an SPM plug-in. ${ }^{24}$ To eliminate interscan variability, $\mathrm{rCBF}$ values were normalized to the $\mathrm{CBF}$ average over the whole brain. In diffusion MRI, ROIs were defined in the posterior limb of the internal capsule using the JHU white matter atlas available in FSL (http://fsl.fmrib.ox.ac. uk/fsl/fslwiki/). ${ }^{38}$
To explore relationships within imaging parameters as well as with systemic blood pressure, the Pearson productmoment coefficient was applied to calculate the correlation coefficient. Patients were divided into 2 subgroups according to the presence or absence of transient neurological deteriorations (TNDs). Both the normalized rCBF and DKI/DTI parameters were compared between these 2 subgroups by using t-tests.

\section{Results}

\section{Patient Characteristics}

The patency of the direct bypass was confirmed in every hemisphere using MR angiography. Transient neurological deteriorations were observed in 6 revascularization surgeries, with aphasia in 2 patients with left-sided surgery, restlessness in 3 patients, and hemisensory disturbance in 2 patients. In 2 patients, postoperative intracerebral hemorrhage was observed. One patient demonstrated severe headache and subsequent hemorrhage on postoperative Day 4 and required evacuation of a hematoma in the right temporal lobe. The other patient demonstrated a right prefrontal subcortical hematoma on postoperative Day 1 but remained asymptomatic. Nine hemispheres demonstrated cortical swelling on T2 and FLAIR images. The TND was not associated with sex (comparisons represent with TND vs without TND; females/males: $5 / 1$ vs $10 / 4$ ), age $(38.4 \pm 10.5$ vs $36.5 \pm 11.0$ years old $)$, clinical presentation (hemorrhage/ischemia: $3 / 3$ vs $6 / 7$ ), conspicuous brain lesion (with/without: $2 / 4$ vs $6 / 8$ ), Suzuki grade (3.0 vs 2.9 [average calculated by converting Roman grades to Arabic grades]), or preoperative mean arterial pressure (MAP; $89.8 \pm 9.9$ vs $93.1 \pm 17.6 \mathrm{~mm} \mathrm{Hg}$ ). Patients were free from apparent neurological deficits at the time of discharge. Table 1 shows the characteristics of the patients.

\section{Topography and Temporal Profile of Cerebral Perfusion}

Figure 1 shows SPECT overlap maps indicating rCBF. An area with increased $\mathrm{rCBF}$ was localized in the basal ganglia on Day $0(\mathrm{p}<0.001)$. On Days $1-2$, the area with increased rCBF extended to the basal ganglia, insula, and portions of the superior temporal and lateral frontal cortices $(\mathrm{p}<0.001)$. Decreased rCBF was also observed in the lateral prefrontal region contralateral to the surgical hemisphere $(\mathrm{p}<0.001)$. On Days $3-6$, a significant rCBF increase was localized in a portion of the basal ganglia as well as in the medial frontal cortex $(\mathrm{p}<0.001)$. On Days $7-8$, an $\mathrm{rCBF}$ increase was observed in the lateral prefrontal cortex $(p<0.001)$. Decreased rCBF was also observed in the lateral prefrontal cortex, occipital lobe, and cerebellum contralateral to the operated hemisphere $(\mathrm{p}<$ 0.001). On Days 11-14, increased $\mathrm{rCBF}$ was localized in the insula $(\mathrm{p}<0.001)$.

Region of interest-based analysis revealed a temporal profile of $\mathrm{rCBF}$ in both the striatum and lateral prefrontal cortex. In the striatum, the rCBF increase was largest on Day 0 and normalized on Days 7-8. In the lateral prefrontal cortex, $\mathrm{rCBF}$ gradually increased over 7-8 postoperative days (Fig. 2). Among 62 measurements, rCBF increases of more than $15 \%$ from baseline were observed in $8.5 \%$ in the lateral prefrontal cortex (range $11.0 \%-25.5 \%$ ) and in $10.2 \%$ in the striatum (range $2.6 \%-19.9 \%$; Fig. 3) 
TABLE 1. Characteristics of the 20 revascularization surgeries performed in 17 adults

\begin{tabular}{|c|c|c|c|c|c|c|c|c|}
\hline Case No. & Sex & Age (yrs) & Clinical Presentation & Side & Brain Lesion & Suzuki Grade & Hypertension & TNDs \\
\hline 1 & M & 44 & TIA & Rt & None & 3 & Yes & No \\
\hline 2 & $\mathrm{~F}$ & 47 & Hemorrhage & Rt & Rt medial temporal \& IVH & 3 & No & No \\
\hline \multirow[t]{2}{*}{3} & $\mathrm{~F}$ & 29 & Hemorrhage & $\mathrm{Lt}$ & IVH & 3 & Yes & Yes \\
\hline & & & - & Rt & - & 3 & - & Yes \\
\hline 4 & $\mathrm{~F}$ & 27 & TIA & $\mathrm{Lt}$ & None & 3 & No & Yes \\
\hline 5 & $\mathrm{~F}$ & 40 & Infarction & $\mathrm{Lt}$ & Rt frontal white matter & 2 & No & No \\
\hline 6 & $\mathrm{~F}$ & 26 & TIA & $\mathrm{Rt}$ & Bilat deep white matter & 3 & No & No \\
\hline 7 & $\mathrm{~F}$ & 34 & TIA & Rt & None & 3 & No & No \\
\hline 8 & $\mathrm{~F}$ & 50 & TIA & Rt & None (SAH) & 3 & No & Yes \\
\hline 9 & M & 38 & Hemorrhage & Lt & Lt frontal deep white matter & 3 & Yes & Yes \\
\hline \multirow[t]{2}{*}{10} & $\mathrm{~F}$ & 33 & Hemorrhage & $\mathrm{Lt}$ & $\mathrm{IVH}$ & 3 & Yes & No \\
\hline & & & - & $\mathrm{Rt}$ & - & 3 & - & No \\
\hline \multirow[t]{2}{*}{11} & $\mathrm{~F}$ & 19 & Hemorrhage & $\mathrm{Lt}$ & Lt basal ganglia & 3 & No & No \\
\hline & & & - & Rt & - & 3 & - & No \\
\hline 12 & $\mathrm{~F}$ & 50 & Hemorrhage & Rt & Rt basal ganglia & 3 & No & No \\
\hline 13 & $\mathrm{~F}$ & 48 & Infarction & Rt & Rt temporal cortical infarction & 3 & No & Yes \\
\hline 14 & $\mathrm{~F}$ & 49 & Asymptomatic & Lt & Lt temporal infarction & 3 & Yes & No \\
\hline 15 & M & 21 & $\mathrm{TIA}$ & $\mathrm{Lt}$ & None & 3 & No & No \\
\hline 16 & M & 29 & TIA & Rt & None & 3 & No & No \\
\hline 17 & M & 46 & TIA & Rt & None & 3 & Yes & No \\
\hline
\end{tabular}

$\mathrm{IVH}=$ intraventricular hemorrhage; $\mathrm{SAH}=$ subarachnoid hemorrhage; $\mathrm{TIA}=$ transient ischemic attack.

\section{Alterations of Brain Microstructure Measured With Diffusion MRI}

The decrease in FA was more pronounced than in any other DTI/DKI parameter. Decreased FA was predominantly observed in the anterior and posterior limbs of the internal capsule and in the pyramidal tracts $(\mathrm{p}<0.001$; Fig. 4). The posterior limb of the internal capsule also showed a decrease in $\mathrm{MK}$ and $\mathrm{AD}$, as well as an increase in RD (p $<0.001$ ). Temporal changes in FA and RD were not synchronized with those in $\mathrm{AD}$, with the greatest changes in FA and RD on Days 1-2 and in AD on Days 3-6 (Fig. 5). There were no significant changes in DKI/DTI parameters in the cortical gray matter.

\section{Correlations Between TNDs and rCBF or Diffusion Parameters}

Preoperative $\mathrm{rCBF}$ was significantly higher in the lateral prefrontal cortex of patients with TNDs than of patients without TNDs $(\mathrm{p}<0.05)$. The highest postoperative rCBF in the striatum of patients with TNDs was significantly greater $(1.28 \pm 0.05)$ than that in patients without TNDs $(1.22 \pm 0.05 ; \mathrm{p}<0.05)$. The highest postoperative rCBF in the lateral prefrontal cortex of patients with TNDs was also significantly greater $(1.11 \pm 0.03)$ than that of patients without TNDs $(1.07 \pm 0.05 ; \mathrm{p}<0.05)$. The lowest postoperative FA of patients with TNDs $(0.36 \pm 0.08)$ was significantly smaller than that of patients without TNDs $(0.44 \pm$ $0.05 ; \mathrm{p}<0.01)$. The lowest postoperative $\mathrm{AD}$ and RK were also significantly smaller in patients with TNDs than in patients without TNDs $(\mathrm{p}<0.05)$. Table 2 summarizes the results of $t$-tests performed on the neuroimaging parameters.
The preoperative MAP showed a modest negative correlation with the $\mathrm{rCBF}$ in the striatum on Days 7-8 ( $\mathrm{r}=$ $-0.55)$. A modest negative correlation between preoperative $\mathrm{rCBF}$ and percent $\mathrm{rCBF}$ changes on Days 7-8 was also observed in the lateral prefrontal cortex $(\mathrm{r}=-0.43)$. A modest negative correlation between preoperative $\mathrm{rCBF}$ and percent $\mathrm{rCBF}$ changes on Days $1-2(\mathrm{r}=-0.47)$ was observed in the striatum.

\section{Discussion}

Combined direct and indirect revascularization surgery in MMD may improve hemodynamic compromise several months after surgery. ${ }^{14}$ However, detrimental effects associated with the surgical procedures can interfere with postoperative management in the initial several weeks. ${ }^{26}$ The current investigation of the process and effects of hyperperfusion and ischemia following combined direct and indirect revascularization sheds valuable light on both the theoretical and clinical understanding of this surgery, its prognosis, and its follow-up.

Additional blood flow through the direct anastomosis was mainly distributed in the basal ganglia immediately after the surgery. Approximately 1 week after surgery, the area of increased $\mathrm{rCBF}$ shifted to the lateral prefrontal cortex. Since chronic ischemia had existed before surgery, the modest increase of blood flow through the direct bypass probably induced an excessive increase in rCBF due to dysautoregulation. The indirect bypass procedure may also contribute to increased rCBF in the lateral prefrontal cortex. As hyperemia is frequently observed beneath subdural hematoma, ${ }^{5}$ brain compression due to swollen mus- 

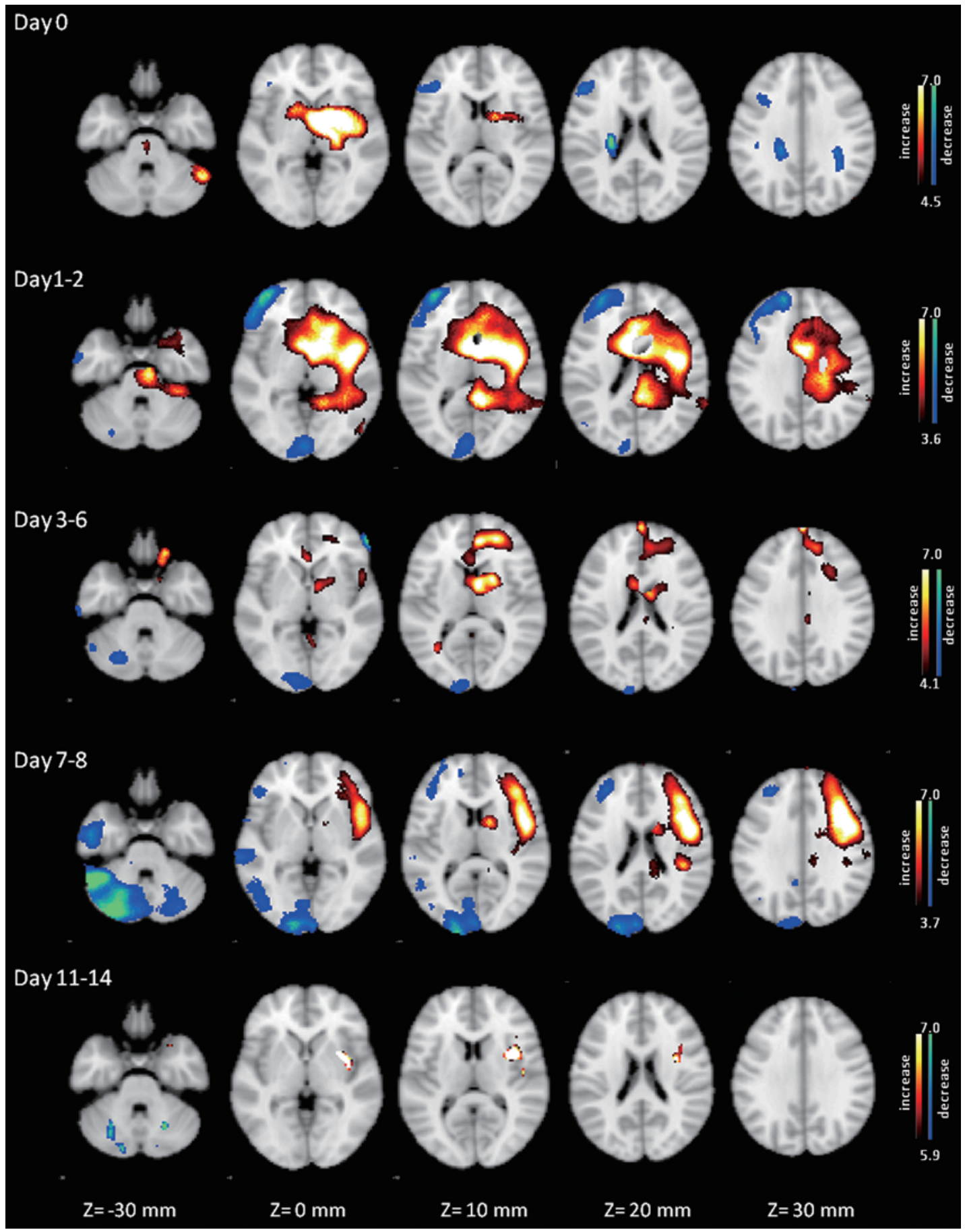

FIG. 1. Topography of rCBF following revascularization surgery in adult MMD. Serial increase and decrease of rCBF maps demonstrate the topography of hyperperfusion. The revascularized hemisphere is set to the right side. Paired t-test models were used in SPM8 software comparing preoperative state on Day 0, Days 1-2, Days 3-6, Days 7-8, and Days 11-14, respectively. Voxels with significant increases in rCBF are shown in red or yellow, whereas voxels with decreases in rCBF are shown in blue or light blue. Color bars represent T values. Lower-threshold T values correspond to a statistical significance of $p<0.001$ with uncorrected family-wise error. An increase of rCBF was observed in the basal ganglia for the first 3 days (Day 0, Days 1-2) after the surgery, followed by a significant increase of rCBF in the lateral prefrontal cortex on Days 7-8. Decreased rCBF was also observed in the lateral prefrontal cortex, occipital lobe, and cerebellum contralateral to the revascularized hemisphere. A decrease of rCBF contralateral to the revascularized hemisphere suggests crossed cerebellar diaschisis due to hyperperfusion. $\mathrm{Z}(\mathrm{mm})=$ coordinates in Montreal Neurological Institute (MNI) space. 

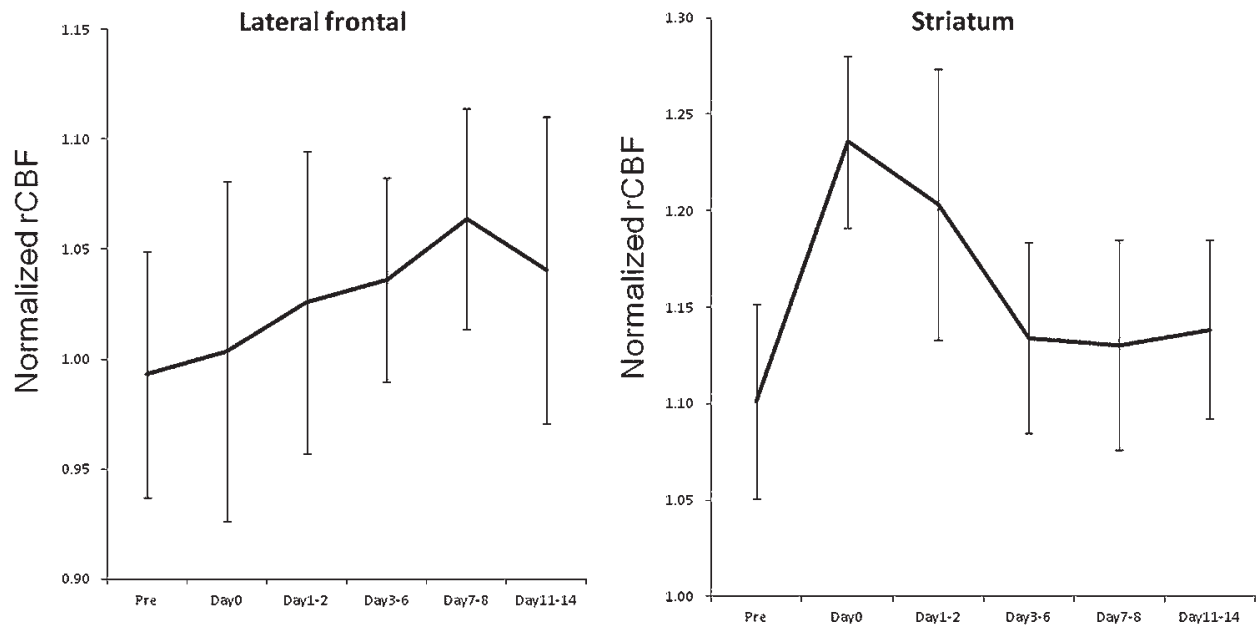

FIG. 2. Temporal profile of rCBF. The rCBF in the lateral prefrontal cortex showed a gradual increase over 7-8 days, whereas the peak rCBF in the striatum was observed on Day 0 and subsequently normalized. Pre $=$ preoperative.
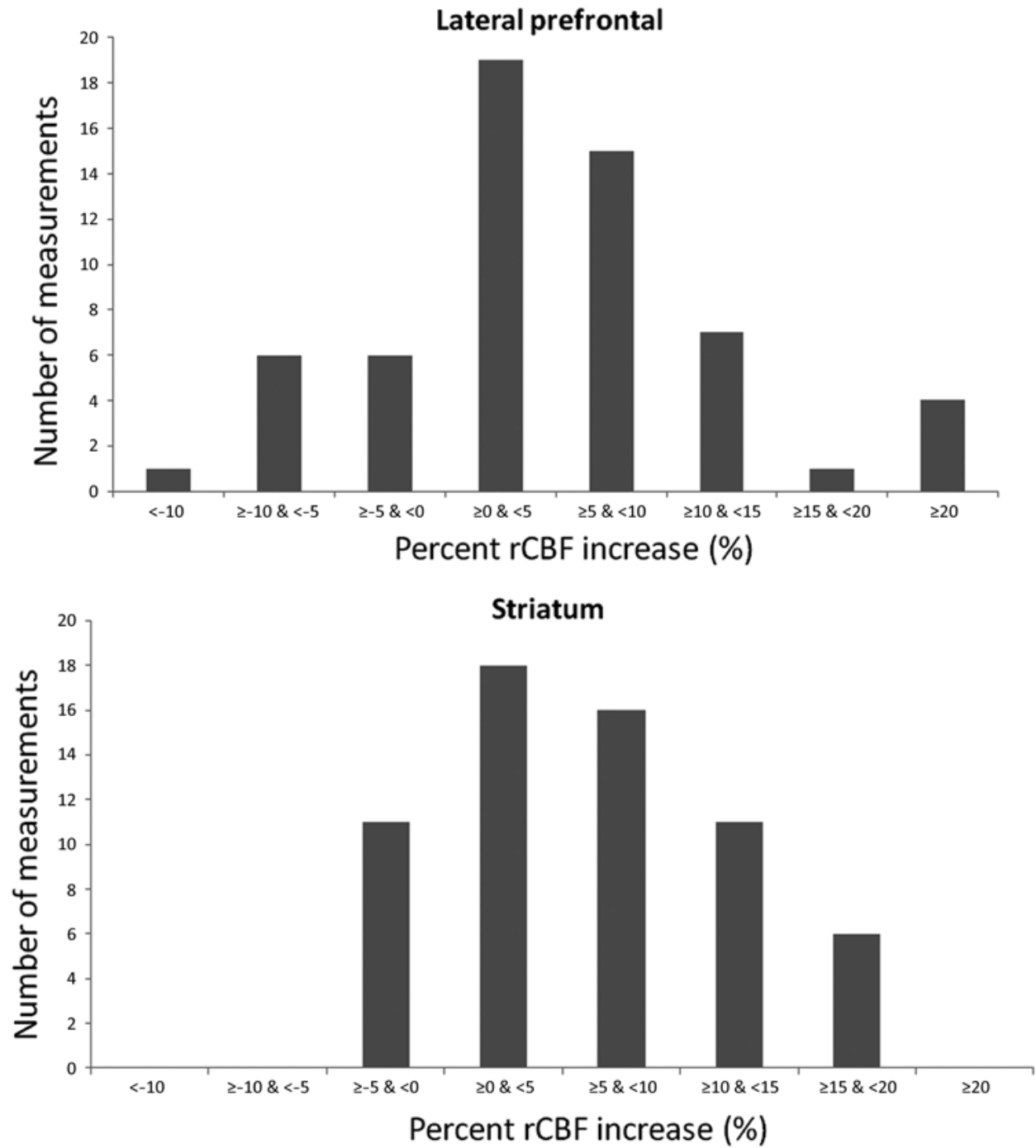

FIG. 3. Histogram of percent changes in $\mathrm{CCBF}$ from baseline. The histogram indicates the number of rCBF measurements out of 62 image acquisitions performed in 17 patients. The majority of the rCBF increase was within $15 \%$ in both the lateral prefrontal cortex and the striatum, showing that hyperperfusion was modest, if present, after revascularization surgery for MMD. 


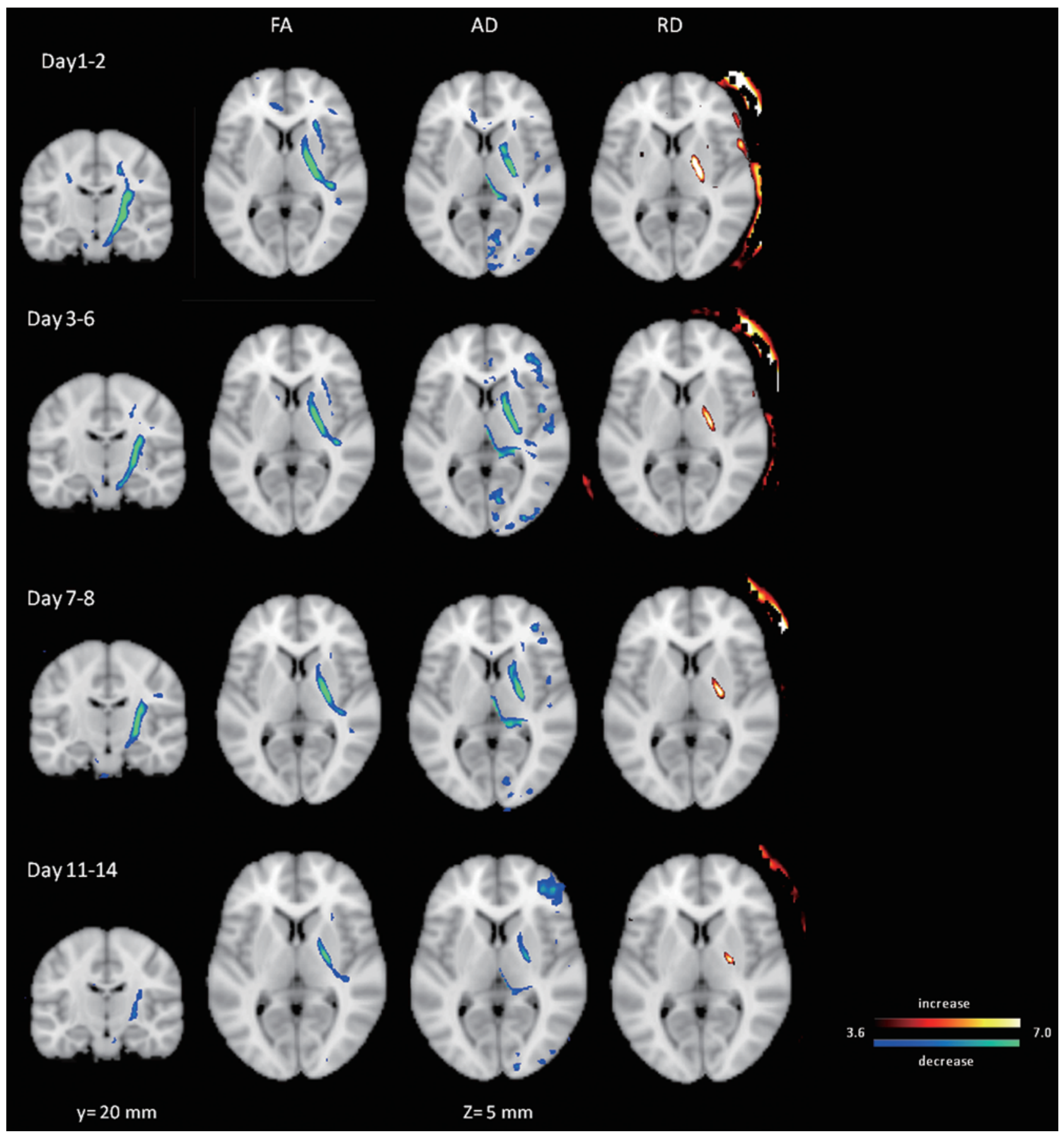

FIG. 4. Serial microstructural changes following revascularization surgery in adult MMD. Changes in diffusion tensor parametersfractional anisotropy (FA), axial diffusivity (AD), and radial diffusivity (RD) - were observed in template space (MNI152_T1_2 mm) available in FSL (http://fsl.fmrib.ox.ac.uk/fsl/fslwiki/). Changes in FA were demonstrated in both coronal $(\mathrm{z}=-16$, coordinates in MNI space) and axial images ( $y=5$, coordinates in MNI space), while changes in both AD and RD were demonstrated in axial images alone ( $y=5$, coordinates in MNI space). Reduced white matter integrity was pronounced in portions of the anterior and posterior limbs of the internal capsule as well as in the pyramidal tract. Decreased AD suggested axonal damage, whereas an increase in RD suggests interstitial edema as well as myelin degeneration.

cles may induce hyperemia during the recovery process. Subdural clots, as well as swollen muscles, in contact with large areas of the cortex can directly affect $\mathrm{rCBF}$ and activate hyperglycolysis. ${ }^{4}$ Furthermore, subclinical seizures can also increase rCBF and metabolism. Immediate post- operative ischemia was also detected, showing decreased rCBF in the lateral prefrontal cortex and occipital lobe contralateral to the revascularized hemisphere. Decreased $\mathrm{rCBF}$ was critical in the hemisphere contralateral to the surgery, as postoperative infarctions were observed in 

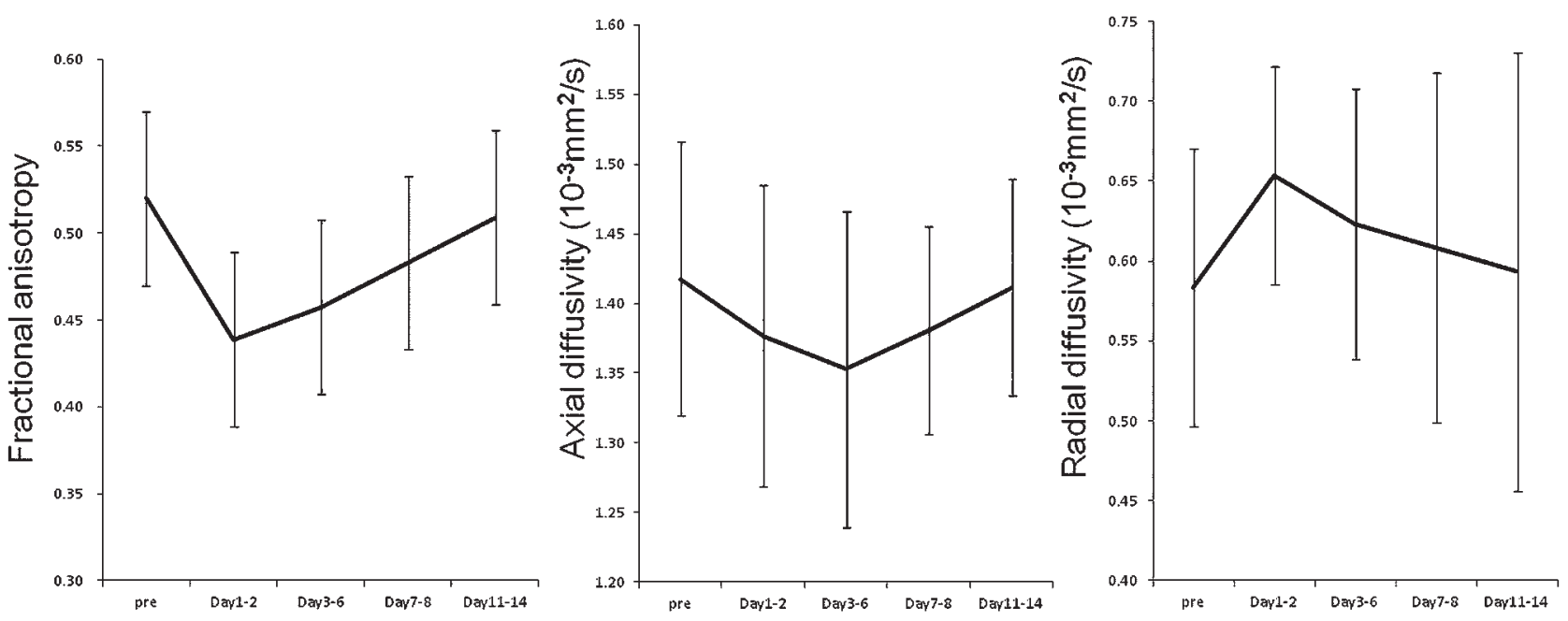

FIG. 5. Temporal profile of diffusion parameter alterations. Serial diffusion MRI revealed significant changes in the DTI parameters: $F A, A D$, and RD. Decreased FA and increased RD were observed on Days 1-2, whereas decreased $A D$ was observed on Days 3-6. This suggests that combined direct and indirect bypass may predominantly impair integrity of white mater tracts for the first several days, probably due to distortion of the brain and interstitial edema. Decreased AD could be interpreted as reduced axonal flow secondary to lateral prefrontal hyperperfusion, which additionally appeared around postoperative Day 5 . These changes in DTI parameters were normalized on postoperative Day 14.

both the prefrontal and posterior cerebral artery territories immediately after the combined direct and indirect bypass..$^{19}$ Decreased rCBF in the cerebellum contralateral to the surgery suggested crossed cerebellar diaschisis. ${ }^{30}$ Interestingly, crossed cerebellar diaschisis, although not conspicuously observed on a patient-by-patient basis, was detected in the voxel-based analysis. ${ }^{15}$

Revascularization surgery may transiently impair subcortical structures without demonstrating white matter lesions on conventional MRI. Changes in diffusion parameters were observed in the pyramidal tract and portions of the anterior limb of the internal capsule. The anterior limb of the internal capsule consists of the corticopontocerebellar pathway, which may be associated with the crossed cerebellar diaschisis found in the voxel-based analysis of rCBF. ${ }^{11}$ None of the patients demonstrated motor weakness, and thus the transient reduction of white matter integrity in the posterior limb of the internal capsule remained asymptomatic.

The DTI parameters are sensitive to changes in the brain's microstructure associated with myelination, axonal membrane integrity, axon and glial density, and coherence of axonal orientation. ${ }^{1}$ In general, decreased FA indicates a decline in the alignment of highly organized cellular structures (axons and myelin). ${ }^{1}$ Observation of decreased FA suggests that white matter integrity decreased along the deep white matter tract. Increased RD occurs due to both myelin degeneration and increased extracellular fluid. ${ }^{31}$ Increased extracellular fluid may be responsible for the reversible increase in RD within short periods of time. Decreased AD may be attributed to axonal impairment. ${ }^{7}$ Although brain shifts or interstitial edema may change DTI parameters such as FA and RD in the first several days, the $\mathrm{AD}$ decreases were more pronounced on Days 3-8. Transient decreases in AD suggest secondary effects of cortical dysfunction, such as reduced axonal transport. ${ }^{3,6}$
A definition of cerebral hyperperfusion has not been established. ${ }^{18,37}$ In the present study, the immediate postsurgical increase in $\mathrm{rCBF}$ remained within $15 \%$ of baseline in the majority of the measurements. There was no clear threshold of the percent rCBF increase required to demonstrate neurological symptoms, which suggested that cortical dysfunction appeared in patients with modest increases in $\mathrm{rCBF}$. In contrast, despite the modest increases in rCBF, gray matter swelling beyond the area of revascularization was observed in approximately half of the studied population, consistent with findings in a previous study. ${ }^{13}$ Thus, modest increases in blood flow could produce breakthrough autoregulation in adult MMD. In such patients, transudation of fluid into the interstitial tissue may induce capillary dysfunction that affects oxygen delivery. ${ }^{29}$

\section{Study Limitations}

There were several limitations to this study. For one, the statistical power to detect group differences depends on the number of studied subjects. Although we were able to detect statistical differences in this study, the number of patients with TNDs was small (6 patients), and our conclusions will require validation using a much larger cohort of patients. In addition, although the rCBF increase was greater in patients with neurological symptoms, the difference was modest. Thus, increased rCBF alone may not be a primary causative factor for the symptomatic hyperperfusion. Furthermore, we cannot rule out the possibility that $\mathrm{rCBF}$ changes associated with TNDs may be at least partially due to worse presurgical states in this subset of patients, despite the fact that these patients did not differ in the clinical characteristics we examined.

There were also limitations inherent to the data acquisition methodology. For example, imaging studies were not performed perfectly in line with the initial protocol because some patients were unable to tolerate image acquisitions during the postoperative period. Region of interest- 
TABLE 2. Comparison of imaging parameters in patients with and without TNDs

\begin{tabular}{|c|c|c|c|c|c|}
\hline \multirow[b]{2}{*}{ Imaging Parameter } & \multicolumn{2}{|c|}{ w/o TNDs } & \multicolumn{2}{|c|}{ w/ TNDs } & \multirow[b]{2}{*}{ p Value* } \\
\hline & Mean & SD & Mean & SD & \\
\hline $\begin{array}{l}\text { Preop rCBF in lateral } \\
\text { prefrontal cortex }\end{array}$ & 0.97 & 0.05 & 1.04 & 0.03 & 0.005 \\
\hline Preop rCBF in striatum & 1.09 & 0.05 & 1.13 & 0.04 & 0.054 \\
\hline $\begin{array}{l}\text { Postop rCBF in lateral } \\
\text { prefrontal cortex }\end{array}$ & 1.07 & 0.05 & 1.11 & 0.03 & 0.047 \\
\hline Postop rCBF in striatum & 1.22 & 0.05 & 1.28 & 0.05 & 0.009 \\
\hline FAt & 0.44 & 0.05 & 0.36 & 0.08 & 0.006 \\
\hline $\mathrm{AD} \dagger$ & 1.36 & 0.07 & 1.27 & 0.12 & 0.023 \\
\hline $\mathrm{RD} \dagger$ & 0.66 & 0.07 & 0.74 & 0.18 & 0.077 \\
\hline MD† & 0.90 & 0.06 & 0.95 & 0.15 & 0.083 \\
\hline MK† & 1.12 & 0.23 & 0.98 & 0.17 & 0.083 \\
\hline AK† & 0.69 & 0.09 & 0.72 & 0.06 & 0.201 \\
\hline RK† & 1.52 & 0.38 & 1.22 & 0.35 & 0.047 \\
\hline
\end{tabular}

based analysis was performed on a complementary basis and yielded consistent results with the voxel-based analysis regarding the temporal profile of $\mathrm{rCBF}$. The range of rCBF increases was smaller than that of a previous study. ${ }^{18}$ This discrepancy may be attributed to the methods of defining ROIs. While the previous study delineated areas of increased $\mathrm{rCBF}$, our study employed a standardized template. A broader range of $\mathrm{rCBF}$ values could be extracted from voxels and resulted in a smaller $\mathrm{rCBF}$ increase compared with the previous study. ${ }^{18}$ However, the ROI in the lateral prefrontal cortex encircled areas of significant change in the rCBF mapping on Days 7-8. Therefore, we believe that the location and the size of the ROIs were less confounded by bias and were optimal. Additionally, postoperative hemorrhage was observed in 2 patients in the present study, with onset on postoperative Day 1 or 4 . Because of the limited number of imaging studies prior to the onset of hemorrhage, a statistical comparison of imaging parameters was not possible between patients with hemorrhage and those without.

Moreover, in the present study, every patient received edaravone. Since free oxygen radical scavengers such as edaravone putatively contain increased permeability due to hyperperfusion, ${ }^{27}$ the frequency of TNDs and the diffusion parameters may have been influenced by edaravone. However, the observed $\mathrm{rCBF}$ increase would not be critically influenced in the voxel-based statistical analysis since a bias toward a lesser impact of hyperperfusion may be expected.

There are some candidate drugs that can alleviate symptomatic hyperperfusion, such as minocyline and the free radical scavenger edaravone. ${ }^{10,36}$ The goal of our study was to identify a potential neuroimaging marker that could track tissue changes associated with hyperperfusion, by which the effect of drug intervention may be more objectively evaluated. The current results, despite the study's limitations, are promising and warrant further validation in a large cohort.

\section{Conclusions}

Perfusion characteristics and microstructural changes following revascularization surgery were analyzed on both a voxel and an ROI basis. A distinct topography of rCBF alterations was observed in each postoperative period. A decreased integrity of white matter was also demonstrated despite a normal appearance of the brain. Increases in $\mathrm{rCBF}$ and changes in diffusion parameters were associated with transient neurological deterioration. This latter association suggests that these tissue structural changes, once further validated in large-scale studies, may have utility as neuroimaging markers during the acute postoperative period following revascularization surgery, potentially enabling better management of hyperperfusion and ischemia associated with MMD.

\section{Acknowledgments}

This study was supported by a grant from the Research Committee on Moyamoya Disease, sponsored by the Ministry of Health, Labor, and Welfare of Japan. This work was also supported by Creation of Innovation Centers for Advanced Interdisciplinary Research Areas Programs, Ministry of Education, Culture, Sports, Science and Technology, Japan.

\section{References}

1. Beaulieu C: The basis of anisotropic water diffusion in the nervous system - a technical review. NMR Biomed 15:435455, 2002

2. Blauwblomme T, Lemaitre H, Naggara O, Calmon R, Kossorotoff M, Bourgeois M, et al: Cerebral blood flow improvement after indirect revascularization for pediatric moyamoya disease: a statistical analysis of arterial spin-labeling MRI.

AJNR Am J Neuroradiol 37:706-712, 2016

3. Budde MD, Xie M, Cross AH, Song SK: Axial diffusivity is the primary correlate of axonal injury in the experimental autoimmune encephalomyelitis spinal cord: a quantitative pixelwise analysis. J Neurosci 29:2805-2813, 2009

4. Bullock R, Butcher SP, Chen MH, Kendall L, McCulloch J: Correlation of the extracellular glutamate concentration with extent of blood flow reduction after subdural hematoma in the rat. J Neurosurg 74:794-802, 1991

5. Chieregato A, Noto A, Tanfani A, Bini G, Martino C, Fainardi E: Hyperemia beneath evacuated acute subdural hematoma is frequent and prolonged in patients with an unfavorable outcome: a Xe-computed tomographic study. Neurosurgery 64:705-718, 2009

6. DeBoy CA, Zhang J, Dike S, Shats I, Jones M, Reich DS, et al: High resolution diffusion tensor imaging of axonal damage in focal inflammatory and demyelinating lesions in rat spinal cord. Brain 130:2199-2210, 2007

7. Fox RJ, Cronin T, Lin J, Wang X, Sakaie K, Ontaneda D, et al: Measuring myelin repair and axonal loss with diffusion tensor imaging. AJNR Am J Neuroradiol 32:85-91, 2011

8. Fujimura M, Kaneta T, Mugikura S, Shimizu H, Tominaga T: Temporary neurologic deterioration due to cerebral hyperperfusion after superficial temporal artery-middle cerebral artery anastomosis in patients with adult-onset moyamoya disease. Surg Neurol 67:273-282, 2007

9. Fujimura M, Mugikura S, Kaneta T, Shimizu H, Tominaga T: Incidence and risk factors for symptomatic cerebral hyperperfusion after superficial temporal artery-middle cerebral 
artery anastomosis in patients with moyamoya disease. Surg Neurol 71:442-447, 2009

10. Fujimura M, Niizuma K, Inoue T, Sato K, Endo H, Shimizu $\mathrm{H}$, et al: Minocycline prevents focal neurological deterioration due to cerebral hyperperfusion after extracranialintracranial bypass for moyamoya disease. Neurosurgery 74:163-170, 2014

11. Fulham MJ, Brooks RA, Hallett M, Di Chiro G: Cerebellar diaschisis revisited: pontine hypometabolism and dentate sparing. Neurology 42:2267-2273, 1992

12. Fushimi Y, Okada T, Takagi Y, Funaki T, Takahashi JC, Miyamoto $\mathrm{S}$, et al: Voxel based analysis of surgical revascularization for Moyamoya disease: pre- and postoperative SPECT studies. PLoS One 11:e0148925, 2016

13. Hamano E, Kataoka H, Morita N, Maruyama D, Satow T, Iihara K, et al: Clinical implications of the cortical hyperintensity belt sign in fluid-attenuated inversion recovery images after bypass surgery for moyamoya disease. $\mathbf{J}$ Neurosurg [epub ahead of print February 19, 2016; DOI: 10.3171/2015.10.JNS151022]

14. Han JS, Abou-Hamden A, Mandell DM, Poublanc J, Crawley AP, Fisher JA, et al: Impact of extracranial-intracranial bypass on cerebrovascular reactivity and clinical outcome in patients with symptomatic moyamoya vasculopathy. Stroke 42:3047-3054, 2011

15. Hokari M, Kuroda S, Simoda Y, Uchino H, Hirata K, Shiga $\mathrm{T}$, et al: Transient crossed cerebellar diaschisis due to cerebral hyperperfusion following surgical revascularization for moyamoya disease: case report. Neurol Med Chir (Tokyo) 52:350-353, 2012

16. Horie N, Morikawa M, Morofuji Y, Hiu T, Izumo T, Hayashi $\mathrm{K}$, et al: De novo ivy sign indicates postoperative hyperperfusion in moyamoya disease. Stroke 45:1488-1491, 2014

17. Houkin K, Kamiyama H, Takahashi A, Kuroda S, Abe H: Combined revascularization surgery for childhood moyamoya disease: STA-MCA and encephalo-duro-arterio-myosynangiosis. Childs Nerv Syst 13:24-29, 1997

18. Kaku Y, Iihara K, Nakajima N, Kataoka H, Fukuda K, Masuoka J, et al: Cerebral blood flow and metabolism of hyperperfusion after cerebral revascularization in patients with moyamoya disease. J Cereb Blood Flow Metab 32:20662075, 2012

19. Kazumata K, Ito M, Tokairin K, Ito Y, Houkin K, Nakayama $\mathrm{N}$, et al: The frequency of postoperative stroke in moyamoya disease following combined revascularization: a single-university series and systematic review. J Neurosurg 121:432440, 2014

20. Kazumata K, Tha KK, Narita H, Ito YM, Shichinohe H, Ito $\mathrm{M}$, et al: Characteristics of diffusional kurtosis in chronic ischemia of adult moyamoya disease: comparing diffusional kurtosis and diffusion tensor imaging. AJNR Am J Neuroradiol [epub ahead of print], 2016

21. Kazumata K, Tha KK, Narita H, Kusumi I, Shichinohe H, Ito $\mathrm{M}$, et al: Chronic ischemia alters brain microstructural integrity and cognitive performance in adult moyamoya disease. Stroke 46:354-360, 2015

22. Kuroda S, Houkin K: Bypass surgery for moyamoya disease: concept and essence of surgical techniques. Neurol Med Chir (Tokyo) 52:287-294, 2012

23. Kuroda S, Houkin K: Moyamoya disease: current concepts and future perspectives. Lancet Neurol 7:1056-1066, 2008

24. Maldjian JA, Laurienti PJ, Burdette JH: Precentral gyrus discrepancy in electronic versions of the Talairach atlas. Neuroimage 21:450-455, 2004

25. Martin AJ, Friston KJ, Colebatch JG, Frackowiak RS: Decreases in regional cerebral blood flow with normal aging. J Cereb Blood Flow Metab 11:684-689, 1991

26. Mukerji N, Cook DJ, Steinberg GK: Is local hypoperfusion the reason for transient neurological deficits after STA-MCA bypass for moyamoya disease? J Neurosurg 122:90-94, 2015

27. Nakamura T, Kuroda Y, Yamashita S, Zhang X, Miyamoto $\mathrm{O}$, Tamiya T, et al: Edaravone attenuates brain edema and neurologic deficits in a rat model of acute intracerebral hemorrhage. Stroke 39:463-469, 2008

28. Nanba T, Ogasawara K, Nishimoto H, Fujiwara S, Kuroda $\mathrm{H}$, Sasaki M, et al: Postoperative cerebral white matter damage associated with cerebral hyperperfusion and cognitive impairment after carotid endarterectomy: a diffusion tensor magnetic resonance imaging study. Cerebrovasc Dis 34:358-367, 2012

29. Østergaard L, Engedal TS, Aamand R, Mikkelsen R, Iversen NK, Anzabi M, et al: Capillary transit time heterogeneity and flow-metabolism coupling after traumatic brain injury. $\mathbf{J}$ Cereb Blood Flow Metab 34:1585-1598, 2014

30. Pantano P, Baron JC, Samson Y, Bousser MG, Derouesne C, Comar D: Crossed cerebellar diaschisis. Further studies. Brain 109:677-694, 1986

31. Riddle A, Dean J, Buser JR, Gong X, Maire J, Chen K, et al: Histopathological correlates of magnetic resonance imagingdefined chronic perinatal white matter injury. Ann Neurol 70:493-507, 2011

32. Saito H, Ogasawara K, Nishimoto H, Yoshioka Y, Murakami T, Fujiwara S, et al: Postoperative changes in cerebral metabolites associated with cognitive improvement and impairment after carotid endarterectomy: a 3T proton MR spectroscopy study. AJNR Am J Neuroradiol 34:976-982, 2013

33. Sakata H, Fujimura M, Mugikura S, Sato K, Tominaga T: Local vasogenic edema without cerebral hyperperfusion after direct revascularization surgery for moyamoya disease. J Stroke Cerebrovasc Dis 24:e179-e184, 2015

34. Tabesh A, Jensen JH, Ardekani BA, Helpern JA: Estimation of tensors and tensor-derived measures in diffusional kurtosis imaging. Magn Reson Med 65:823-836, 2011

35. Uchino H, Kuroda S, Hirata K, Shiga T, Houkin K, Tamaki $\mathrm{N}$ : Predictors and clinical features of postoperative hyperperfusion after surgical revascularization for moyamoya disease: a serial single photon emission $\mathrm{CT} /$ positron emission tomography study. Stroke 43:2610-2616, 2012

36. Uchino H, Nakayama N, Kazumata K, Kuroda S, Houkin $\mathrm{K}$ : Edaravone reduces hyperperfusion-related neurological deficits in adult moyamoya disease: a historical control study. Stroke [epub ahead of print], 2016

37. van Mook WN, Rennenberg RJ, Schurink GW, van Oostenbrugge RJ, Mess WH, Hofman PA, et al: Cerebral hyperperfusion syndrome. Lancet Neurol 4:877-888, 2005

38. Wakana S, Jiang H, Nagae-Poetscher LM, van Zijl PC, Mori $\mathrm{S}$ : Fiber tract-based atlas of human white matter anatomy. Radiology 230:77-87, 2004

\section{Disclosures}

The authors report no conflict of interest concerning the materials or methods used in this study or the findings specified in this paper.

\section{Author Contributions}

Conception and design: Kazumata. Acquisition of data: Tha, Uchino, Shiga. Analysis and interpretation of data: Kazumata, Tha. Drafting the article: Kazumata. Critically revising the article: Tha, Ito, Shichinohe, Nakayama, Abumiya. Approved the final version of the manuscript on behalf of all authors: Kazumata. Statistical analysis: Kazumata. Study supervision: Kazumata.

\section{Correspondence}

Ken Kazumata, Department of Neurosurgery, Hokkaido University Graduate School of Medicine, North 15 West 7, Kita, Sapporo 060-8638, Japan. email: kazumata@med.hokudai.ac.jp. 